\title{
THE BLACK HOLE FORMATION PROBABILITY
}

\author{
Drew Clausen, Anthony L. Piro, and Christian D. Ott \\ TAPIR, Walter Burke Institute for Theoretical Physics, California Institute of Technology, Mailcode 350-17, \\ Pasadena, CA 91125, USA; dclausen@tapir.caltech.edu \\ Received 2014 June 18; accepted 2014 November 27; published 2015 January 28
}

\begin{abstract}
A longstanding question in stellar evolution is which massive stars produce black holes (BHs) rather than neutron stars (NSs) upon death. It has been common practice to assume that a given zero-age main sequence (ZAMS) mass star (and perhaps a given metallicity) simply produces either an NS or a BH, but this fails to account for a myriad of other variables that may effect this outcome, such as spin, binarity, or even stochastic differences in the stellar structure near core collapse. We argue that instead a probabilistic description of NS versus $\mathrm{BH}$ formation may be better suited to account for the current uncertainties in understanding how massive stars die. We present an initial exploration of the probability that a star will make a $\mathrm{BH}$ as a function of its ZAMS mass, $P_{\mathrm{BH}}\left(M_{\mathrm{ZAMS}}\right)$. Although we find that it is difficult to derive a unique $P_{\mathrm{BH}}\left(M_{\mathrm{ZAMS}}\right)$ using current measurements of both the BH mass distribution and the degree of chemical enrichment by massive stars, we demonstrate how $P_{\mathrm{BH}}\left(M_{\mathrm{ZAMS}}\right)$ changes with these various observational and theoretical uncertainties. We anticipate that future studies of Galactic BHs and theoretical studies of core collapse will refine $P_{\mathrm{BH}}\left(M_{\mathrm{ZAMS}}\right)$ and argue that this framework is an important new step toward better understanding $\mathrm{BH}$ formation. A probabilistic description of $\mathrm{BH}$ formation will be useful as input for future population synthesis studies that are interested in the formation of X-ray binaries, the nature and event rate of gravitational wave sources, and answering questions about chemical enrichment.
\end{abstract}

Key words: black hole physics - galaxies: abundances - nuclear reactions, nucleosynthesis, abundances - stars: massive - supernovae: general

\section{INTRODUCTION}

It is currently not known which massive stars result in black holes (BHs) rather than neutron stars (NSs). There is convincing evidence for stellar mass BHs from X-ray binaries throughout our galaxy (Remillard \& McClintock 2006), so it is clear BHs must be a possible endpoint of stellar evolution in some situations. The inferred masses of these observed BHs indicate a distribution of $\approx 4.5-15 M_{\odot}$ that is strikingly distinct from the typical masses of NSs of $\approx 1.3-2 M_{\odot}$ (Bailyn et al. 1998; Özel et al. 2010; Farr et al. 2011). The apparent lack of BH masses from $\approx 4.5 M_{\odot}$ down to the maximum mass of NSs may be an important clue about the types of stars or situations that lead to $\mathrm{BH}$ formation.

Recently, Kochanek (2014) argued that this separation of masses may be naturally understood if the loosely bound hydrogen shell of massive stars is lost prior to $\mathrm{BH}$ formation. This could be due to a low-energy shock triggered by a reduction of the gravitational mass from neutrino emission during the proto-NS phase which precedes stellar-mass $\mathrm{BH}$ formation (Nadezhin 1980; Lovegrove \& Woosley 2013; Piro 2013). In this case, the BH mass would be determined by the remaining helium core mass prior to core collapse. Pre-explosion imaging of corecollapse supernovae ( $\mathrm{SNe}$ ) suggests zero-age main sequence (ZAMS) progenitor masses $8 \lesssim M_{\text {ZAMS }} \lesssim 17 M_{\odot}$ (Smartt et al. 2009) for standard Type II-P SNe that are thought to produce NSs. If this upper mass limit implies that unsuccessful explosions and $\mathrm{BH}$ formation occur for $M_{\mathrm{ZAMS}} \gtrsim 17 M_{\odot}$, then the typical helium core mass of these stars naturally explains the mass scale of the stellar mass BHs we observe (Kochanek 2014). This is in contrast to stellar model calculations that artificially drive explosions and consider $\mathrm{BH}$ formation via fallback of outer core and envelope material (e.g., Zhang et al. 2008, and references therein). These studies find some $\mathrm{BH}$ masses in a range of $\approx 2.5-4.5 M_{\odot}$, contrary to what is observed (but see the recent work of Ugliano et al. 2012, who found little fallback in any successful explosion).

On the theoretical side there is also much uncertainty in determining which massive stars produce BHs and what the typical BH masses should be. Studies by Timmes et al. (1996), Fryer (1999), Fryer \& Kalogera (2001), Heger et al. (2003), Eldridge \& Tout (2004), Zhang et al. (2008), O'Connor \& Ott (2011), Belczynski et al. (2012), and Ugliano et al. (2012) attempt to connect the outcomes of stellar collapse to the progenitor ZAMS mass and metallicity. In particular, O'Connor \& Ott (2011) quantified whether or not a star was likely to produce a successful explosion via a compactness parameter ( $\propto M / R(M)$, for some representative maximum NS mass $M$ ), with a higher compactness implying a star was more likely to form a $\mathrm{BH}$. An interesting feature of the compactness elucidated by this work and, subsequently in more detail by Sukhbold \& Woosley (2014), was that it is not a monotonic function of the ZAMS mass; it can be significantly higher or lower depending on the mass range of interest, and can even abruptly change between models that are relatively close in ZAMS mass.

If the compactness is this sensitive to the details of stellar evolution, then macroscopic differences in massive stars, whether it be metallicity, rotation rate, mass loss events, or binarity, likely have a profound impact on whether a given star forms a BH or NS. Couch \& Ott (2013) have shown that precollapse perturbations from convective shell burning can increase the strength of turbulence behind the stalled SN shock and thus aid neutrino-driven explosions. If this depends on the magnitude and stochastic spatial structure of the perturbations, then even small stochastic differences from event to event may alter whether neutrino heating can successfully revive the stalled shock and power a SN. Altogether, it is clear that any simple prescription that attempts to connect $M_{\text {ZAMS }}$ directly to NS or $\mathrm{BH}$ formation will be insufficient. This has motivated us to 
consider a different paradigm for thinking about $\mathrm{BH}$ formation: a probabilistic description for $\mathrm{BH}$ formation.

In the following study we explore whether $\mathrm{BH}$ formation can be described as a probabilistic process. Instead of assuming that a given $M_{\text {ZAMS }}$ (or even that a given $M_{\text {ZAMs }}$ plus metallicity) will either produce a $\mathrm{BH}$ or not, we attempt to infer what probability function $P_{\mathrm{BH}}\left(M_{\mathrm{ZAMS}}\right)$ is implied by the observed distribution of $\mathrm{BH}$ masses. We then investigate the implications of this probability function, from the enrichment of heavy elements due to the explosion or collapse of massive stars to the connection to the compactness of massive stars from stellar modeling.

In Section 2, we describe the observed BH mass distribution and invert this distribution to produce two example probability functions for $\mathrm{BH}$ formation. We attempt to refine these $\mathrm{BH}$ formation probability functions using nucleosynthetic constraints on $\mathrm{BH}$ formation in Section 3. In Section 4, we discuss the $\mathrm{BH}$ formation probabilities and explore their possible origin. Caveats involving the nature of mass loss in massive stars are also explored in Section 4. Finally, our key results are summarized in Section 5.

\section{THE BLACK HOLE MASS FUNCTION}

Before we make use of the observed $\mathrm{BH}$ mass distribution, it is important to consider how it may be affected by systematic errors or selection biases and whether this should have any impact on our conclusions. Özel et al. (2010) and Farr et al. (2011) carried out independent, Bayesian analyses of 16 and 15, respectively, black hole X-ray transients (BHXRTs) to determine the underlying $\mathrm{BH}$ mass distribution. In both studies the inferred intrinsic $\mathrm{BH}$ mass distribution peaks in the range 5-7 $M_{\odot}$ and declines rapidly at larger masses. Farr et al. (2011) explored the functional form of the distribution using Bayesian model selection, and found that it was best described as a power law. Alternatively, motivated by the theoretical work of Fryer \& Kalogera (2001), Özel et al. (2010) assumed that the functional form of the $\mathrm{BH}$ mass distribution was a decaying exponential. Even though Farr et al. (2011) found that the data favor a power law mass distribution, we use a fit to the normalized, weighted BH mass distribution of Özel et al. (2010) because it is easily incorporated into our mathematical formalism. The fit is given in the appendix to Özel et al. (2012). We have confirmed that using a power-law distribution does not significantly change the results presented below.

Observational uncertainties could have a larger impact on our work than these two different models. Kreidberg et al. (2012) argued that the orbital inclinations of the BHXRTs used to construct the $\mathrm{BH}$ mass distribution may be systematically underestimated. If these systems had larger inclinations, then the measured BH masses would be systematically overestimated. However, as Kreidberg et al. (2012) pointed out, more data are needed to determine whether this is actually the case.

One selection bias in the BHXRT sample that has potential implications for our work is the simple fact that these BHs are all in binaries. We are therefore attempting to use the mass distribution of BHs specific to binary systems to make broader conclusions about the probability that any given star will form a $\mathrm{BH}$ or not. It could be that this observed $\mathrm{BH}$ mass distribution is a product of the unique evolutionary channel that produces BHXRTs, and not a generic outcome of stellar evolution and core collapse. In fact, Farr et al. (2011) showed that the masses of BHs found in BHXRTs were not consistent with being drawn at random from a $\mathrm{BH}$ mass distribution constructed from a sample that included BHs from both BHXRTs and high mass X-ray binaries. This discrepancy may suggest that binary evolutionary processes are influencing these mass distributions, or it may also indicate that the high mass X-ray binaries have $\mathrm{BH}$ masses that do not reflect their mass at birth. As there is little hope of measuring the mass distribution of single BHs, we elect to make use of the mass function presented in Özel et al. (2012) in our study despite these issues. Furthermore, we focus on just BHXRT rather than include the high mass X-ray binaries because Farr et al. (2011) showed that the low and high mass systems are drawn from separate populations.

\subsection{Inverting the BH Mass Distribution}

Using the $\mathrm{BH}$ mass distribution discussed above, we derive the probability that a star of given ZAMS mass will produce a $\mathrm{BH}$. The problem of inverting the $\mathrm{BH}$ mass distribution is underdetermined because the $\mathrm{BH}$ formation probability is a priori a free function (i.e., it has an infinite number of free parameters). Since our aim is to introduce the concept of probabilistic $\mathrm{BH}$ formation, we will impose several restrictive assumptions to make the problem tractable. Accordingly, we caution the reader that while the solutions presented below are consistent with current, weak theoretical and observational constraints, they are only examples of a much larger set of possible solutions.

Inferring a probability function for $\mathrm{BH}$ formation $P_{\mathrm{BH}}$ $\left(M_{\text {ZAMS }}\right)$ from the $\mathrm{BH}$ mass distribution requires two primary model inputs. First, we need to specify an initial mass function (IMF), which sets the mass distribution of ZAMS stars. We assume the IMF given in Salpeter (1955), $\Psi\left(M_{\text {ZAMS }}\right) d M_{\text {ZAMS }} \propto$ $M_{\text {ZAMS }}^{-2.35} d M_{\text {ZAMS }}$. Several studies of stellar populations in a range of environments have confirmed that stars with $M_{\text {ZAMS }} \gtrsim 3 M_{\odot}$ are drawn from a distribution with this power law slope (Bastian et al. 2010 and references therein).

The second component we need is a function that relates a star's ZAMS mass to the mass of the $\mathrm{BH}$ it produces $M_{\mathrm{BH}}\left(M_{\mathrm{ZAMS}}\right)$. Many of the aspects of massive star evolution that have motivated us to consider a probabilistic description of $\mathrm{BH}$ formation could also produce stochasticity in the $M_{\mathrm{ZAMS}}-M_{\mathrm{BH}}$ relationship. Accounting for this would require us to specify a distribution function for $M_{\mathrm{BH}}$ given $M_{\mathrm{ZAMS}}$. For this initial exploration of the $\mathrm{BH}$ formation probability, we feel it is reasonable to restrict our calculations to a simple relationship between ZAMS mass and BH mass. This condition is equivalent to assuming that the stochasticity in $M_{\mathrm{BH}}\left(M_{\mathrm{ZAMS}}\right)$ is folded into $P_{\mathrm{BH}}$. Further, in the discussion and calculations that follow, we will consider only solar metallicity stars. The impact of our assumed $M_{\mathrm{ZAMS}}-M_{\mathrm{BH}}$ relationship on the shape of $P_{\mathrm{BH}}$ will be explored in Section 4.1.

As discussed in Section 1, there is evidence that the star's helium core mass sets the scale of the BH mass. The main physical reason why this is an attractive picture is that when the star becomes a red giant, the hydrogen envelope is so loosely bound that it can easily become removed in a number of different ways. In particular, the energy carried away by neutrinos during the post-bounce, pre-BH-formation phase is sufficient to unbind the hydrogen envelopes of stars with ZAMS masses in the range 15-25 $M_{\odot}$ (Nadezhin 1980; Lovegrove \& Woosley 2013; Piro 2013). Even for stars just outside of this mass range, the envelopes have sufficiently low binding energies that it is plausible that this mechanism could operate between $\sim 12 M_{\odot}$ and $\sim 30 M_{\odot}$.

In addition, significant portions of the hydrogen envelopes could also be ejected during pre-SN eruptions (e.g., Smith et al. 


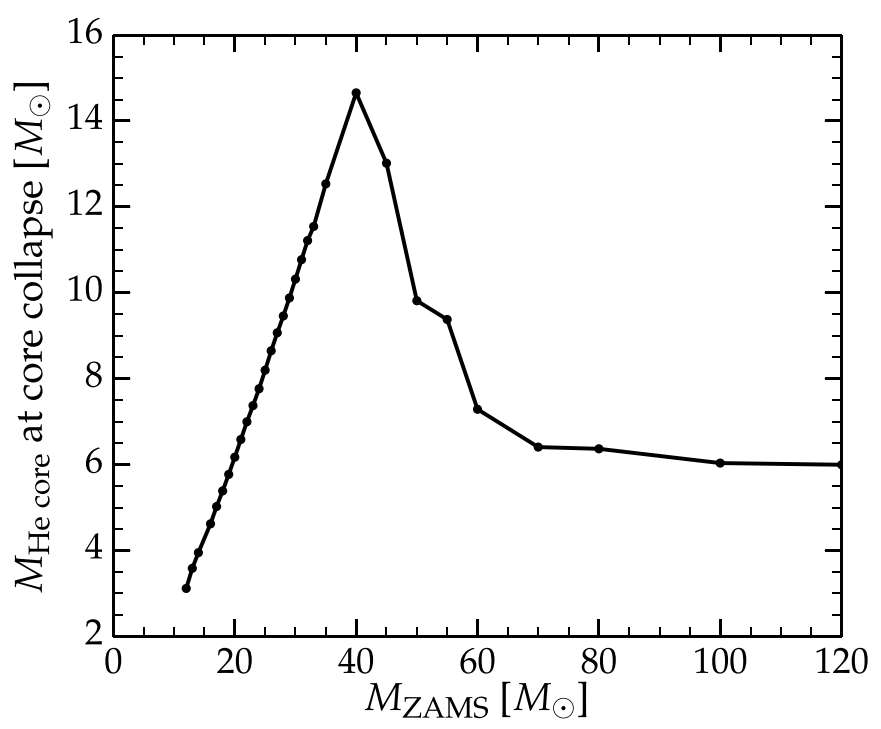

Figure 1. Helium core mass vs. ZAMS mass from the stellar evolution models of Woosley \& Heger (2007). These models are of non-rotating stars with solar metallicity. In this work, we assume that if a star collapses into a $\mathrm{BH}$, the mass of the $\mathrm{BH}$ is equal to the mass of the star's He core, i.e., $M_{\mathrm{BH}}\left(M_{\mathrm{ZAMS}}\right) \equiv M_{\mathrm{He}}$ core $\left(M_{\mathrm{ZAMS}}\right)$. We also ignore the distinction between gravitational and baryonic masses.

2011; Smith \& Arnett 2014; Shiode \& Quataert 2014). These more energetic ( $~ 10^{48} \mathrm{erg}$ ) precursor events may be necessary to remove the more tightly bound envelopes of stars in the ZAMS mass range $\sim 30-40 M_{\odot}$. However, stellar winds will remove all but about $1 M_{\odot}$ of these stars' envelopes before core collapse (Woosley \& Heger 2007). Even if the star is unable to shed this final portion of the envelope, the resulting $\mathrm{BH}$ mass would only increase by $5 \%-10 \%$. Within the current understanding of mass loss, the most massive stars, with $M_{\text {ZAMS }} \gtrsim 40 M_{\odot}$, are WolfRayet stars with extreme winds that will completely remove the envelope before core collapse.

Given all of these reasons, it seems unlikely that a significant portion of the hydrogen envelope will be incorporated into the $\mathrm{BH}$ produced by a star in the entire mass range $12-120 M_{\odot}$. Accordingly, we choose to use the He core mass at the onset of core collapse as the resulting BH mass, i.e., $M_{\mathrm{BH}}\left(M_{\mathrm{ZAMS}}\right) \equiv$ $M_{\text {He core }}\left(M_{\text {ZAMS }}\right)$ (Kochanek 2014; Burrows 1987). The He core masses are taken from the non-rotating, solar metallicity stellar evolution models presented in Woosley \& Heger (2007), and are shown in Figure 1. We define the boundary of the He core as the location where the $\mathrm{H}$ mass fraction drops below $1 \%$ and extract the He core masses from models at the pre-SN stage. Under this definition, the binding energy of the hydrogen envelope is in the range $10^{47-48} \mathrm{erg}$; low enough that the processes described above are able to remove it.

Other potential functions we could have used for $M_{\mathrm{BH}}\left(M_{\mathrm{ZAMS}}\right)$ include the total mass of the star at the moment of core collapse or the results from detailed numerical models that look at fallback during a successful SN. The former case is unable to reproduce the observed $\mathrm{BH}$ mass distribution because these massive stars will produce $\mathrm{BHs}$ that are more massive than any BHXRTs. The latter scenario is favored, e.g., by Fryer (1999), Fryer \& Kalogera (2001), Zhang et al. (2008), and Fryer et al. (2012). These authors suggest that BH formation could occur via fallback accretion in successful, but weak explosions. However, Dessart et al. (2010) point out that this requires an unlikely fine-tuning of explosion energy to envelope binding energy. This point is corroborated by the results of Ugliano et al. (2012), who find very little fallback in their successful explosions of solar-metallicity stars. Coupled with studies of the NS mass distribution (Pejcha et al. 2012), there seems to be a strong indication that fallback does not play a large roll in most SN explosions, which may have important implications for future studies of SN explosion mechanisms. Motivated by these studies, we do not consider BH formation via fallback after a successful explosion in this exploratory work. Subsequent studies should explore the effect of fallback even if there currently is little consensus about its relevance.

As can be seen in Figure 1, the function that relates a $\mathrm{BH}$ mass to a ZAMS mass, $M_{\mathrm{ZAMS}}\left(M_{\mathrm{BH}}\right)$, is double valued. BHs with $M_{\mathrm{BH}}>6 M_{\odot}$ are potentially produced by stars in two separate ZAMS mass ranges, one below $40 M_{\odot}$ and one above. We therefore must take special consideration of these two mass ranges, and we relate the initial stellar population, described by the IMF, to the descendent $\mathrm{BH}$ population using

$$
\begin{aligned}
\int_{M_{1}}^{M_{2}} & \Psi\left(M_{\mathrm{ZAMS}}\right) P_{\mathrm{BH}}\left(M_{\mathrm{ZAMS}}\right) d M_{\mathrm{ZAMS}} \\
& +\int_{M_{3}}^{M_{4}} \Psi\left(M_{\mathrm{ZAMS}}\right) P_{\mathrm{BH}}\left(M_{\mathrm{ZAMS}}\right) d M_{\mathrm{ZAMS}} \\
& =\int_{M_{\mathrm{BH}, 1}}^{M_{\mathrm{BH}, 2}} \Psi_{\mathrm{BH}}\left(M_{\mathrm{BH}}\right) d M_{\mathrm{BH}},
\end{aligned}
$$

where $\Psi_{\mathrm{BH}}\left(M_{\mathrm{BH}}\right)$ is the $\mathrm{BH}$ mass distribution, $M_{\mathrm{BH}, 1}=$ $M_{\mathrm{BH}}\left(M_{1}\right)=M_{\mathrm{BH}}\left(M_{4}\right)$, and $M_{\mathrm{BH}, 2}=M_{\mathrm{BH}}\left(M_{2}\right)=M_{\mathrm{BH}}\left(M_{3}\right)$. The first term on the left-hand side of Equation (1) accounts for the BHs in the mass range $M_{\mathrm{BH}, 1}$ to $M_{\mathrm{BH}, 2}$ that are produced by stars in the ZAMS mass range $M_{1}$ to $M_{2}$, where $M_{1}<M_{2} \leqslant 40 M_{\odot}$. The second term on the left-hand side then describes the contribution to this $\mathrm{BH}$ mass range from stars with ZAMS masses $M_{4}>M_{3}>40 M_{\odot}$. Our goal is to determine the fraction of stars in these ZAMS mass ranges that are needed to collapse into $\mathrm{BHs}$ to account for the number of BHs expected in the mass range $M_{\mathrm{BH}, 1}$ to $M_{\mathrm{BH}, 2}$, given the observed $\mathrm{BH}$ mass distribution. We interpret this fraction as the probability that a star of given ZAMS mass will form a $\mathrm{BH}$, $P_{\mathrm{BH}}\left(M_{\mathrm{ZAMS}}\right)$.

We solve for $P_{\mathrm{BH}}\left(M_{\mathrm{ZAMS}}\right)$ by recasting Equation (1) as a system of two coupled differential equations for $d P_{\mathrm{BH}, \text { low }} / d M_{\mathrm{BH}}$ and $d P_{\mathrm{BH}, \text { high }} / d M_{\mathrm{BH}}$. Here the subscripts low (high) correspond to the probability function for stars with ZAMS mass below (above) $40 M_{\odot}$. We numerically integrate the system over the BH mass range 6.0-14.66 $M_{\odot}$. The lower limit is the mass of a BH produced by a star with $M_{\text {ZAMS }}=120 M_{\odot}$, the most massive star considered in our study. The corresponding ZAMS mass below $40 M_{\odot}$ (i.e., the low mass star that produces a $6.0 M_{\odot}$ $\mathrm{BH})$ is $19.22 M_{\odot}$. The upper limit is set by the maximum He core mass in the Woosley \& Heger (2007) models, which corresponds to a ZAMS mass of $40 M_{\odot}$. Therefore, the integration over $M_{\mathrm{BH}}$ is equivalent to integrating inward in $M_{\text {ZAMS }}$ from the low and high mass ends, simultaneously.

As an additional constraint, we assume that $P_{\mathrm{BH}}\left(M_{\mathrm{ZAMS}}\right)$ is continuous. This means that the value of $P_{\mathrm{BH}}\left(40 M_{\odot}\right)$ must be the same whether it was approached from the low mass or high mass side. Although continuity is not at all required, if it were not included the problem of finding $P_{\mathrm{BH}}\left(M_{\mathrm{ZAMS}}\right)$ would become highly degenerate since small ranges of mass with $M_{\text {ZAMS }}<40 M_{\odot}$ could be exchanged with $M_{\text {ZAMS }}>40 M_{\odot}$ (and vice versa) and still match the overall $\mathrm{BH}$ mass distribution. Since our main goal is to illustrate $P_{\mathrm{BH}}\left(M_{\mathrm{ZAMS}}\right)$ for the first time, 

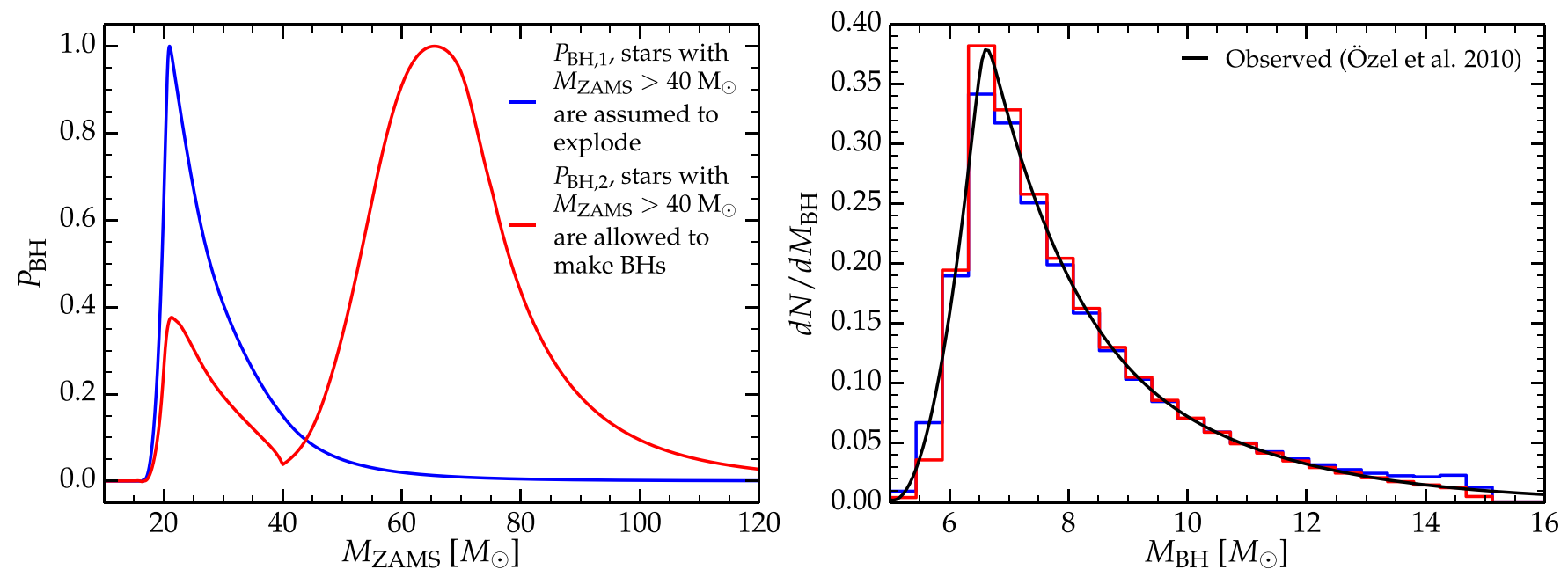

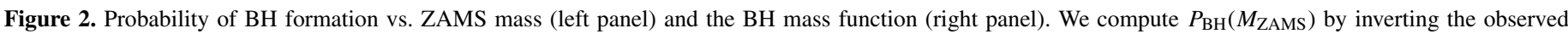

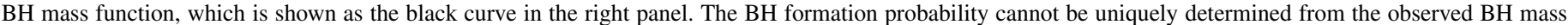

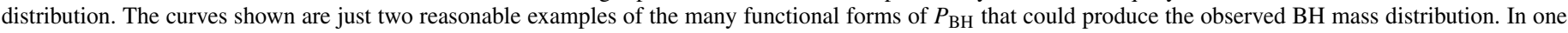

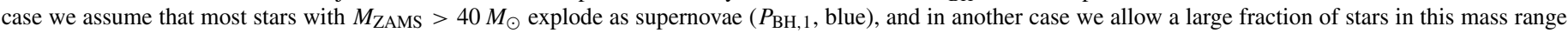

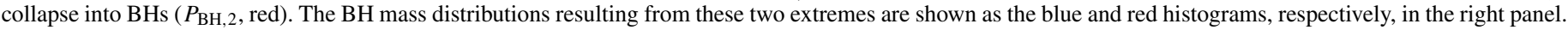

we feel it is reasonable to use this restriction of continuity to have a tractable problem until future observations or theoretical calculations provide reasons to consider more complicated functional forms for $P_{\mathrm{BH}}\left(M_{\mathrm{ZAMS}}\right)$.

To solve the equations, we use the shooting method to adjust the boundary conditions $P_{\mathrm{BH}, \text { low }}\left(19.22 M_{\odot}\right)$ and $P_{\mathrm{BH}, \text { high }}\left(120 M_{\odot}\right)$ until integration yields $P_{\mathrm{BH}, \text { low }}\left(40 M_{\odot}\right)=$ $P_{\mathrm{BH}, \text { high }}\left(40 M_{\odot}\right)$. Once a matching solution is identified, we continue to integrate $d P_{\mathrm{BH}, \text { low }} / d M_{\mathrm{BH}}$ down to $M_{\mathrm{BH}}=5.0 M_{\odot}$. These low mass BHs are only produced by stars with $M_{\text {ZAMS }}<$ $19.22 M_{\odot}$, so there is no contribution from the high mass stars. In our calculations, we set $\Psi_{\mathrm{BH}}\left(M_{\mathrm{BH}}<5 M_{\odot}\right)=0$. Observations suggest that these low mass BHs are extremely rare, with $\Psi_{\mathrm{BH}}\left(5 M_{\odot}\right)$ a factor of 150 lower than the mass distribution's peak at $M_{\mathrm{BH}}=6.6 M_{\odot}$.

Finally, we normalize the probability function so that its maximum value is one. As such, the $\mathrm{BH}$ formation probabilities presented here are upper limits because it is possible that $P_{\mathrm{BH}}\left(M_{\mathrm{ZAMS}}\right)<1$ for all stars. In the following section, we discuss the results of these calculations.

\subsection{The BH Formation Probability Function}

As we discussed above, the underdetermined nature of the problem prevents us from inferring a unique $\mathrm{BH}$ formation probability function from the observed $\mathrm{BH}$ mass distribution. Our assumed $M_{\mathrm{ZAMS}}-M_{\mathrm{BH}}$ relationship imposes another degeneracy because BHs of a given mass can sometimes be produced by stars with two different ZAMS masses (see Figure 1). Although the IMF dictates that there will be drastically different numbers of stars in these ZAMS mass ranges, the value of $P_{\mathrm{BH}}$ at these masses could, in principle, differ by a similar factor and remove the IMF's influence. This leads to an ambiguity in $P_{\mathrm{BH}}\left(M_{\mathrm{ZAMS}}\right)$. Due to this degeneracy we compute examples of $P_{\mathrm{BH}}$ under two extreme scenarios. The results are illustrated in Figure 2. The two BH formation probability functions are shown in the left panel, and the resulting $\mathrm{BH}$ mass distributions are compared with the fit to the observed $\mathrm{BH}$ mass distribution in the right panel.

In one extreme, we assume that most stars with $M_{\text {ZAMS }}>$ $40 M_{\odot}$ successfully explode as SNe and produce NSs. To solve for $P_{\mathrm{BH}}\left(M_{\mathrm{ZAMS}}\right)$ in this case, we require that $d P_{\mathrm{BH}} / d M_{\mathrm{ZAMS}} \leqslant$ 0 for $M_{\mathrm{ZAMS}}>40 M_{\odot}$. In this scenario, the $\mathrm{BH}$ formation probability increases rapidly above $M_{\text {ZAMS }}=17 M_{\odot}$, peaks around $21 M_{\odot}$, and then gradually declines for larger $M_{\mathrm{ZAMS}}$, dropping to zero for $M_{\text {ZAMS }} \gtrsim 70 M_{\odot}$. We label this probability function $P_{\mathrm{BH}, 1}$. For the second extreme, we do not impose any restrictions on stars with $M_{\text {ZAMS }}>40 M_{\odot}$. The resulting probability function exhibits two peaks, one around $21 M_{\odot}$ and a second, broad peak at $65.5 M_{\odot}$. We label this $\mathrm{BH}$ formation probability function $P_{\mathrm{BH}, 2}$.

Under the assumptions that we imposed to produce examples of the $\mathrm{BH}$ formation probability, these two extremes illustrate the minimum $\left(P_{\mathrm{BH}, 1}\right)$ and maximum $\left(P_{\mathrm{BH}, 2}\right)$ contribution to the $\mathrm{BH}$ population from stars with $M_{\mathrm{ZAMS}}>40 M_{\odot}$. In each case, there is a peak in $P_{\mathrm{BH}}$ near $M_{\mathrm{ZAMS}}=20 M_{\odot}$, suggesting that the $\mathrm{BH}$ mass distribution requires that some stars of this mass collapse into BHs. In our models, the lowest mass BHs $\left(M_{\mathrm{BH}} \sim 5 M_{\odot}\right)$ can only be produced by stars in this mass range. On the other hand, the observed BH mass distribution can be reproduced with or without a peak in the probability function at high $M_{\text {ZAMS }}$. Because the shape of the $\mathrm{BH}$ formation probability function is not well constrained by the $\mathrm{BH}$ mass function, we explore whether $P_{\mathrm{BH}, 1}$ and $P_{\mathrm{BH}, 2}$ are consistent with other observational constraints on $\mathrm{BH}$ formation.

\section{NUCLEOSYNTHESIS}

Stellar nucleosynthesis has been established as a means of probing BH formation (Twarog \& Wheeler 1982; Maeder 1992; Brown \& Woosley 2013). If a star fails to explode, most of the nuclear burning products created during its lifetime become part of a $\mathrm{BH}$ instead of enriching the interstellar medium (ISM). Thus, constraints can be placed on $\mathrm{BH}$ formation by comparing observed abundance patterns to the nucleosynthetic yields of model stellar populations that assume different $\mathrm{BH}$ formation scenarios. Traditionally, $\mathrm{BH}$ formation was assumed to occur above a particular ZAMS mass, $M_{\mathrm{BH}}^{\star}$. That is, all stars with $M_{\text {ZAMS }}>M_{\mathrm{BH}}^{\star}$ produce BHs, and all stars in the range $8 M_{\odot} \lesssim M_{\text {ZAMS }}<M_{\mathrm{BH}}^{\star}$ explode and produce NSs. Maeder (1992) found that the observed ratio of helium enrichment to metal enrichment was best matched by models that had $M_{\mathrm{BH}}^{\star}$ 
between $20 M_{\odot}$ and $25 M_{\odot}$. Models by Brown \& Woosley (2013) showed that similar cutoff masses could produce material of solar composition, and further suggested that accounting for uncertainties in stellar mass loss and nuclear reaction rates could drive $M_{\mathrm{BH}}^{\star}$ to $18 M_{\odot}$. However, the authors also found that the solar abundances were well matched by models with a cutoff mass as large as $120 \mathrm{M}_{\odot}$. In this section we test the $\mathrm{BH}$ formation probability functions computed above against these nucleosynthetic constraints.

The nucleosynthesis of massive stars is delivered to the ISM by two mechanisms, SN explosions and winds. Accordingly, we calculate the mass $\left(m_{i}\right)$ of isotope $i$ produced by a stellar population using

$$
\begin{aligned}
m_{i}=\int_{12 M_{\odot}}^{120 M_{\odot}} & {\left[1-P_{\mathrm{BH}}(M)\right] \Psi(M) E_{i}(M) d M } \\
& +\int_{12 M_{\odot}}^{120 M_{\odot}} \Psi(M) W_{i}(M) d M,
\end{aligned}
$$

where $E_{i}(M)$ and $W_{i}(M)$ give the mass of isotope $i$ ejected in the SN explosion and wind, respectively, of a star of ZAMS mass $M$. The values of $E_{i}(M)$ and $W_{i}(M)$ were taken from the yield table presented in Brown \& Woosley (2013). The integration limits in Equation (2) were set by the range of models included in the Brown \& Woosley (2013) table. The first integral on the right hand side of Equation (2) accounts for the explosive yields. This material is only released to the ISM if the star explodes. The second integral accounts for the material lost in winds before core collapse, material which enriches the ISM whether or not the SN explosion fails.

The nucleosynthetic yields resulting from the example $\mathrm{BH}$ formation probabilities computed in Section 2.2 are shown in Figure 3. In our analysis, we examine the mass fractions of isotopes relative to ${ }^{12} \mathrm{C}$ because ${ }^{12} \mathrm{C}$ is ejected primarily in the winds of the most massive stars. Accordingly, the ${ }^{12} \mathrm{C}$ yield is insensitive to which stars explode, and comparing the abundances of other isotopes relative to ${ }^{12} \mathrm{C}$ highlights differences in the explosive yields arising from the different $\mathrm{BH}$ formation scenarios. However, stellar mass loss physics is poorly understood. Thus, we caution that this property of ${ }^{12} \mathrm{C}$ may be a consequence of the treatment of wind mass loss in the Woosley \& Heger (2007) models.

The scenarios considered here, $P_{\mathrm{BH}, 1}$ and $P_{\mathrm{BH}, 2}$, produce nearly identical nucleosynthetic yields. For most isotopes, the relative abundances change by $\lesssim 10 \%$ when we switch from $P_{\mathrm{BH}, 1}$ to $P_{\mathrm{BH}, 2}$. The largest changes occur amongst the intermediate mass elements. Significant amounts of ${ }^{32} \mathrm{~S},{ }^{36} \mathrm{Ar}$, and ${ }^{40} \mathrm{Ca}$ are produced in stars with $M_{\text {ZAMS }} \sim 20 M_{\odot}$. Roughly $60 \%$ of these stars explode as $\mathrm{SNe}$ and eject this material into the ISM when we assume that $\mathrm{BH}$ formation is described by $P_{\mathrm{BH}, 2}$. In the case of $P_{\mathrm{BH}, 1}$, the explosion fails in almost all of these stars and the material falls into BHs. The different $\mathrm{BH}$ formation probability functions result in changes of $13.6 \%$, $14.1 \%$, and $14.0 \%$ in the relative abundances of ${ }^{32} \mathrm{~S},{ }^{36} \mathrm{Ar}$, and ${ }^{40} \mathrm{Ca}$, respectively. While these intermediate mass elements are sensitive to the different $\mathrm{BH}$ formation scenarios described by $P_{\mathrm{BH}, 1}$ and $P_{\mathrm{BH}, 2}$, the changes in the expected yields are too small to determine whether one scenario is favored over the other, given the uncertainty in massive star nucleosynthetic yields.

We also compare the yields resulting from the $\mathrm{BH}$ formation probabilities computed in this work with those resulting from the traditional cutoff mass scenario. Figure 4 compares the relative abundances produced in a calculation that uses $P_{\mathrm{BH}, 1}$ with those produced when we assume $M_{\mathrm{BH}}^{\star}=25 M_{\odot}$. For most elements, there is fairly good agreement between the two cases. However, the relative abundances of two groups of isotopes vary significantly between these scenarios. First, the yields of the $\alpha$-elements ${ }^{16} \mathrm{O},{ }^{20} \mathrm{Ne}$, and ${ }^{24} \mathrm{Mg}$ change by approximately $50 \%$. In the cutoff mass scenario, the ISM is not enriched by the explosive yields from stars with $M_{\text {ZAMS }}>25 M_{\odot}$. While ${ }^{16} \mathrm{O}$, ${ }^{20} \mathrm{Ne}$, and ${ }^{24} \mathrm{Mg}$ are produced in stars with $M_{\text {ZAMS }}<25 M_{\odot}$, a considerable fraction of the total, IMF-weighted production of these isotopes occurs in stars with ZAMS masses between $30 M_{\odot}$ and $50 M_{\odot}$. In the $P_{\mathrm{BH}, 1}$ case, many of the stars in this mass range undergo successful explosions, so the $\alpha$-elements that they produce are delivered to the ISM, boosting these isotopes' relative abundances. Stars in this same mass range are also responsible for the substantially different yields predicted for the $s$-process elements. In the Woosley \& Heger (2007) models, ${ }^{70} \mathrm{Ge},{ }^{76} \mathrm{Se},{ }^{86} \mathrm{Sr}$, and ${ }^{87} \mathrm{Sr}$ are primarily synthesized in stars in the ZAMS mass range $25-50 M_{\odot}$. Therefore, the relative abundances of these isotopes increase by roughly $70 \%$ when we compute the yields using $P_{\mathrm{BH}, 1}$ instead of using $M_{\mathrm{BH}}^{\star}=25 M_{\odot}$. Despite these differences, the nucleosynthesis produced by a population of stars that form BHs according to the probability function $P_{\mathrm{BH}, 1}$ matches the production of a population in which all stars with $M_{\text {ZAMS }}>25 M_{\odot}$ form BHs, within the factor of two uncertainty suggested by Brown \& Woosley (2013).

Rather than selecting a single value of $M_{\mathrm{BH}}^{\star}$ and computing the relative abundances of several ions, a complementary comparison between these different $\mathrm{BH}$ formation scenarios can be made by selecting specific abundance ratios and varying $M_{\mathrm{BH}}^{\star}$. Figure 5 shows how the ratios of ${ }^{20} \mathrm{Ne} /{ }^{16} \mathrm{O}$ and ${ }^{28} \mathrm{Si} /{ }^{16} \mathrm{O}$ change as $M_{\mathrm{BH}}^{\star}$ increases from $13 M_{\odot}$ to $90 M_{\odot}$. The values of these ratios from calculations using the $\mathrm{BH}$ formation probability functions $P_{\mathrm{BH}, 1}$ and $P_{\mathrm{BH}, 2}$ are plotted as well. The probability functions predict similar ${ }^{20} \mathrm{Ne} /{ }^{16} \mathrm{O}$ ratios to the cutoff mass scenario with $M_{\mathrm{BH}}^{\star} \sim 35 M_{\odot}$. For the ${ }^{28} \mathrm{Si} /{ }^{16} \mathrm{O}$ ratio, the probability models agree with the cutoff case at a lower value of $M_{\mathrm{BH}}^{\star} \sim 17 M_{\odot}$. Although the values of $M_{\mathrm{BH}}^{\star}$ differ, both are in reasonable agreement with previous limits on the $\mathrm{BH}$ formation cutoff mass. These discrepant values of $M_{\mathrm{BH}}^{\star}$ are equivalent to the inconsistencies in the relative abundances of some isotopes discussed above.

Perhaps in the future these methods can be used to differentiate between the $\mathrm{BH}$ formation scenarios, but with the present levels of theoretical and observational uncertainty in massive star nucleosynthetic yields it is not possible to determine which model best matches the data. However, given the reasonable agreement between the yields and the equivalent values of $M_{\mathrm{BH}}^{\star}$, we can conclude that the illustrative $\mathrm{BH}$ formation probability functions $P_{\mathrm{BH}, 1}$ or $P_{\mathrm{BH}, 2}$ are consistent with previous, weak nucleosynthetic constraints on $\mathrm{BH}$ formation.

\section{SOURCES OF UNCERTAINTY AND THE PHYSICAL ORIGIN OF $P_{\mathrm{BH}}$}

We have proposed that $\mathrm{BH}$ formation can be described as a probabilistic process and have used the observed distribution of $\mathrm{BH}$ masses to explore the probability that a star of given $M_{\text {ZAMS }}$ will produce a $\mathrm{BH}, P_{\mathrm{BH}}\left(M_{\mathrm{ZAMS}}\right)$. We cannot uniquely determine the functional form of $P_{\mathrm{BH}}\left(M_{\mathrm{ZAMS}}\right)$, instead we have inferred two example $\mathrm{BH}$ formation probability functions that are consistent with the observed $\mathrm{BH}$ mass distribution and nucleosynthetic constraints on $\mathrm{BH}$ production. We next examine 


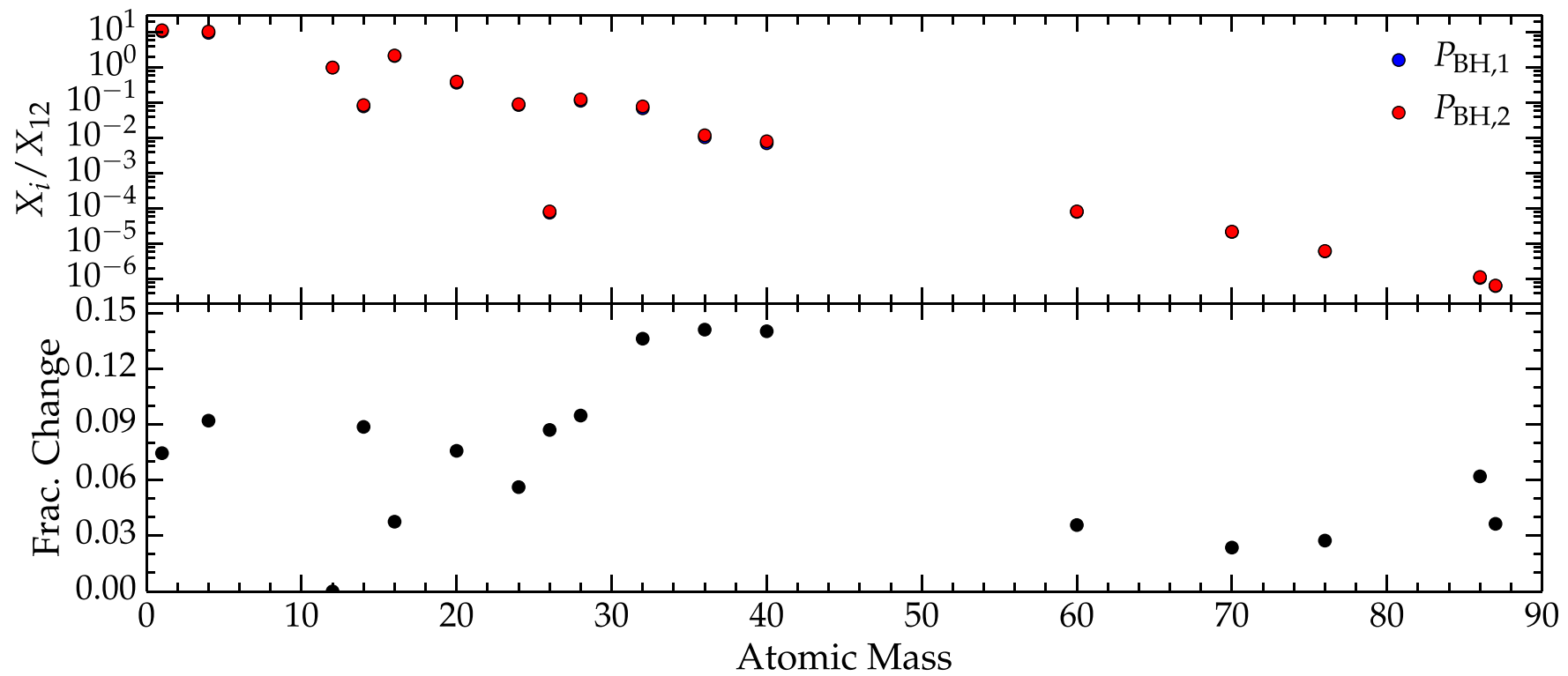

Figure 3. Nucleosynthetic yields resulting from the BH formation probabilities shown in Figure 2. The top panel shows the mass fractions of various isotopes relative to ${ }^{12} \mathrm{C}$ vs. atomic mass. The relative abundances are shown for $P_{\mathrm{BH}, 1}$ (blue), and $P_{\mathrm{BH}, 2}$ (red). The yields are very similar in these two scenarios, so the red symbols completely cover the blue symbols for most isotopes. We have chosen to show the abundances relative to ${ }^{12} \mathrm{C}$ because this isotope is primarily ejected by the winds of massive stars and is therefore insensitive to the functional form of $P_{\mathrm{BH}}$. Comparing the abundances of other isotopes to the nearly constant ${ }^{12} \mathrm{C}$ abundance accentuates differences in the yields. The lower panel shows the fractional change in the relative abundances when the different $\mathrm{BH}$ formation probabilities are assumed. For most isotopes, the change was $<10 \%$. The relative abundances of the intermediate mass isotopes ${ }^{32} \mathrm{~S},{ }^{36} \mathrm{Ar}$, and ${ }^{40} \mathrm{Ca}$ are most sensitive to which $P_{\mathrm{BH}}$ function is used and change by $13.6 \%, 14.1 \%$, and $14.0 \%$, respectively.

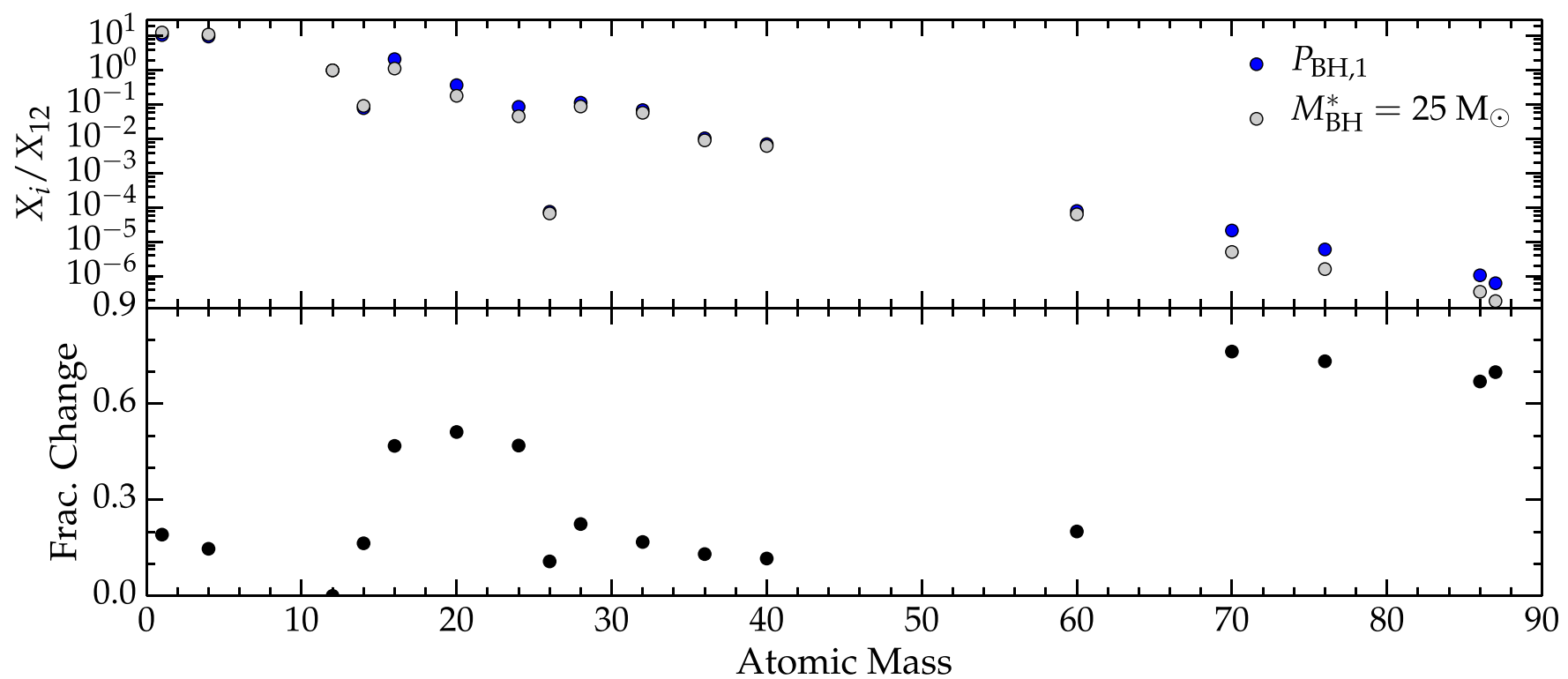

Figure 4. Same as Figure 3, but comparing the yields for $P_{\mathrm{BH}, 1}$ (blue) and a traditional BH formation scenario in which all stars with $M_{\mathrm{ZAMS}}>25 M_{\odot}$ collapse into BHs (gray). The production of many isotopes changes by less than $20 \%$ when the different $\mathrm{BH}$ formation probabilities are used. Stars with $M_{\text {ZAMS }}>25 M_{\odot}$ produce significant amounts of the $\alpha$-elements ${ }^{16} \mathrm{O},{ }^{20} \mathrm{Ne}$, and ${ }^{24} \mathrm{Mg}$, as well as the $s$-process elements ${ }^{70} \mathrm{Ge},{ }^{76} \mathrm{Se},{ }^{86} \mathrm{Sr},{ }^{87} \mathrm{Sr}$. In the $P_{\mathrm{BH}, 1}$ scenario, these products are delivered to the ISM when stars above $25 M_{\odot}$ explode, leading to an increase in the relative abundances of these isotopes over the traditional $\mathrm{BH}$ formation scenario.

the assumptions made above, discuss the physical origin of $P_{\mathrm{BH}}$, and investigate the impact of mass loss on $\mathrm{BH}$ progenitors.

\subsection{The $M_{\mathrm{ZAMS}}-M_{B H}$ Relationship}

Computing $P_{\mathrm{BH}}\left(M_{\mathrm{ZAMS}}\right)$ requires a relationship between $M_{\text {ZAMS }}$ and $M_{\mathrm{BH}}$. In Section 2.1, we argued that the helium core mass at the onset of core collapse was a reasonable estimate for $M_{\mathrm{BH}}$. There are a number of assumptions incorporated into generating a helium core mass. In our calculations, we extracted the helium core masses from the models of Woosley \& Heger (2007). Initial conditions and several of the physical processes in these stellar evolution calculations can influence the final mass of helium cores, including rotation rate, metallicity, and mass loss mechanisms. Stellar evolution models that include rotation typically produce more massive helium cores than nonrotating models (e.g., Heger et al. 2000; Meynet \& Maeder 2000). The magnitude of the increase is sensitive to the treatment of rotationally induced mixing, but the He core can grow by as much as $30 \%$. At lower metallicity, a star of given $M_{\text {ZAMS }}$ produces a $10 \%-20 \%$ more massive helium core than the solar metallicity stars modeled by Woosley \& Heger (2007). Additionally, wind mass loss and metallicity are closely linked. 

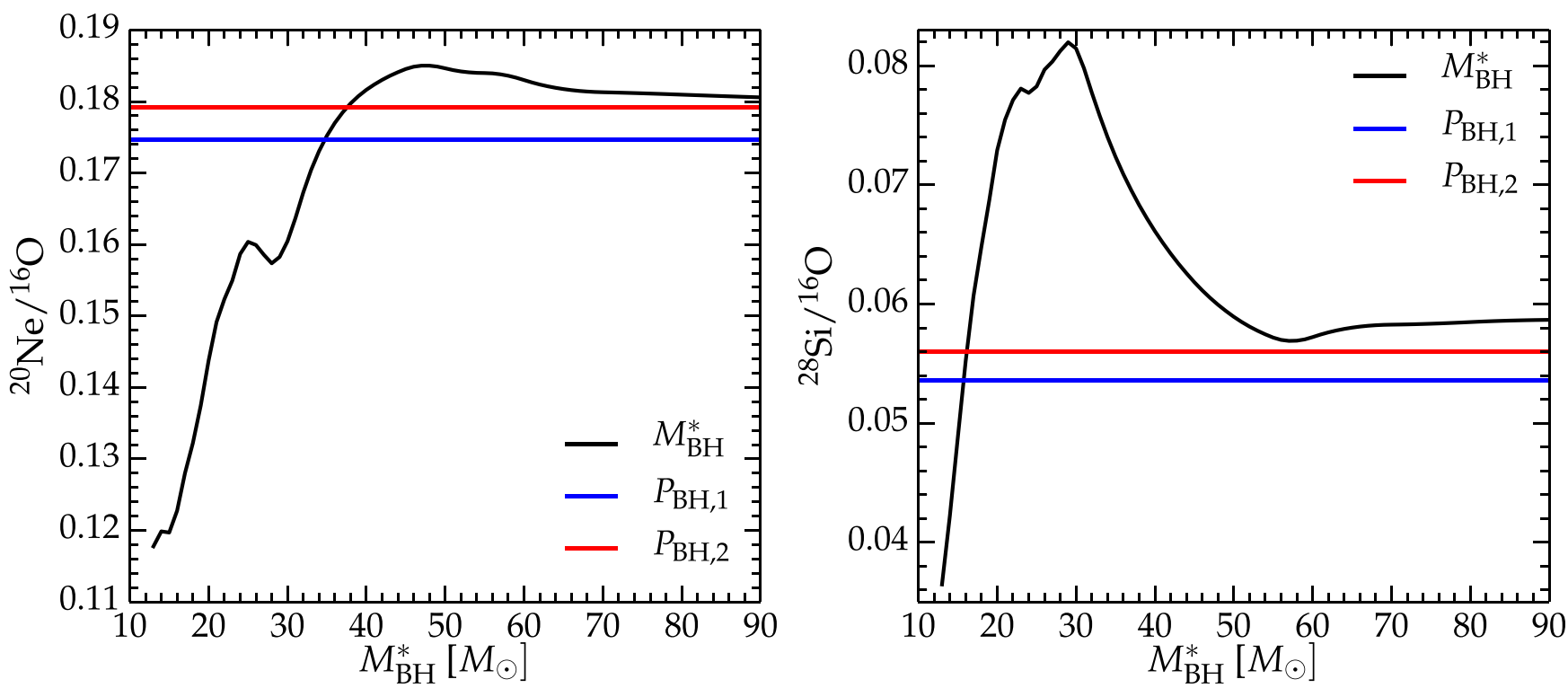

Figure 5. Mass fraction of ${ }^{20} \mathrm{Ne}$ (left panel) and ${ }^{28} \mathrm{Si}$ (right panel) relative to ${ }^{16} \mathrm{O}$ vs. the threshold ZAMS mass for BH production. The relative abundances of ${ }^{20} \mathrm{Ne}$ and ${ }^{28} \mathrm{Si}$ resulting from calculations that use $\mathrm{BH}$ formation probabilities $P_{\mathrm{BH}, 1}(M)$ (blue) and $P_{\mathrm{BH}, 2}(M)$ (red) are also shown. These figures illustrate an observational test that could differentiate between the traditional $\mathrm{BH}$ formation scenario and the $\mathrm{BH}$ formation scenarios explored here. Namely, the relative abundances of different isotopes will imply different threshold masses for $\mathrm{BH}$ formation. In the example shown, the values of ${ }^{20} \mathrm{Ne} /{ }^{16} \mathrm{O}$ calculated with the BH formation probabilities $P_{\mathrm{BH}, 1}(M)$ and $P_{\mathrm{BH}, 2}(M)$ are consistent with $M_{\mathrm{BH}}^{\star} \sim 35 M_{\odot}$. On the other hand, the ${ }^{28} \mathrm{Si} /{ }^{16} \mathrm{O}$ ratio implies a lower value of $M_{\mathrm{BH}}^{\star} \sim 17 M_{\odot}$.

At low metallicity, the opacity in the envelope drops, greatly reducing the rate of radiation driven mass loss in stars with $M_{\text {ZAMS }}>40 M_{\odot}$. As a result of this, the helium cores of high mass, low metallicity stars will be significantly more massive than those considered in our calculations. The envelope also stays much more compact than in the solar-metallicity case and red supergiants become rarer. Because of this, Zhang et al. (2008) argued that fallback could be copious in low-metallicity progenitor stars, which, however, would be inconsistent with the $\mathrm{BH}$ mass distribution observed today.

Rotation and metallicity-dependent mass loss, among other effects, will complicate the $M_{\mathrm{BH}}\left(M_{\text {ZAMS }}\right)$ relationship and drive it away from the simple relationship assumed in Section 2.1. Figure 6 illustrates the possible impact of these $\mathrm{BH}$ mass variations on the $\mathrm{BH}$ formation probability function. To generate this form of $P_{\mathrm{BH}}$, we assume that the mass of a $\mathrm{BH}$ produced by a star of given ZAMS mass is drawn from a normal distribution. The mean of the distribution is the final helium core mass from the Woosley \& Heger (2007) models and its full width at half maximum (FWHM) is $0.5 M_{\text {He core }}\left(M_{\text {ZAMS }}\right)$. Introducing another free parameter, the width of the BH mass distribution, requires that we impose an additional restriction when inverting the BH mass distribution. Namely, we assume that all stars that produce a helium core of given mass will collapse into BHs with equal probability. This is in contrast to Section 2.1, where we treated $P_{\mathrm{BH} \text {,low }}$ and $P_{\mathrm{BH}, \mathrm{hi}}$ as completely independently quantities.

The inferred $\mathrm{BH}$ formation probability function is similar to $P_{\mathrm{BH}, 2}$, however the peaks are broadened and shifted toward one another. Furthermore, in this example both peaks reach the same height, $P_{\mathrm{BH}}=1$. This is a consequence of our assumption that the $\mathrm{BH}$ formation probability is determined by the helium core mass, independent of the ZAMS mass. Finally, we find that the $\mathrm{BH}$ formation scenario described by this form of $P_{\mathrm{BH}}$ is consistent with the weak nucleosynthetic constraints discussed in Section 3.

The BH formation probability function shown in Figure 6 accounts for a moderate amount of stochastic variation in $\mathrm{BH}$

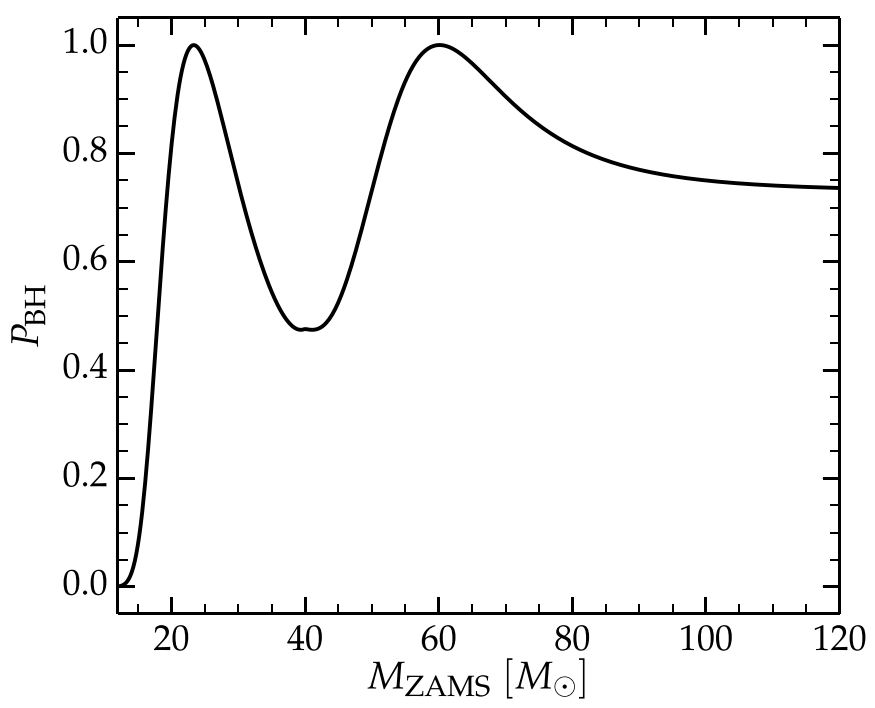

Figure 6. Example probability of $\mathrm{BH}$ formation vs. ZAMS mass assuming stochasticity in the $M_{\mathrm{ZAMS}}-M_{\mathrm{BH}}$ relationship. The $\mathrm{BH}$ masses are drawn from a normal distribution centered on the final helium core mass (see Figure 1) with a FWHM of $0.5 M_{\mathrm{He}}$ core. Considering a range of possible $\mathrm{BH}$ masses broadens the peaks in $P_{\mathrm{BH}}$ and shifts the first (second) peak to larger (smaller) ZAMS mass. Our method for inferring the $\mathrm{BH}$ formation probability from the $\mathrm{BH}$ mass distribution forces the peaks to be of equal height. This form of $P_{\mathrm{BH}}$ is also consistent with nucleosynthetic constraints on $\mathrm{BH}$ formation.

mass. It does not capture extreme variations, e.g., very massive $\mathrm{BHs}$ produced by high-mass, low-metallicity stars. Furthermore, it still assumes that the scale of the BH mass is set by the helium core mass and not, for example, the strength of the explosion and fallback onto the proto-NS. In conclusion, because the $M_{\mathrm{ZAMS}}-M_{\mathrm{BH}}$ relationship is not well understood, it is possible that $P_{\mathrm{BH}}\left(M_{\mathrm{ZAMS}}\right)$ differs from the functions inferred in this work. Nevertheless, the $\mathrm{BH}$ formation probability functions shown here are plausible representations within the current understanding of stellar evolution and a useful first step toward 
introducing the new paradigm of probabilistic $\mathrm{BH}$ formation that we are advocating.

\subsection{Uncertainty in the BH Mass Distribution}

Next, we consider how statistical uncertainties in the observed $\mathrm{BH}$ mass distribution impact the $\mathrm{BH}$ formation probability functions that we have inferred. To investigate the propagation of the statistical uncertainties, we use a parameterized version of the $\mathrm{BH}$ mass function that assumes the form of the distribution is a decaying exponential

$$
\Psi\left(M_{\mathrm{BH}}\right)=\left\{\begin{array}{ll}
\frac{e^{M_{c} / M_{\text {scale }}}}{M_{\text {scale }}} \exp \left[-\frac{M_{\mathrm{BH}}}{M_{\text {scale }}}\right] & M_{\mathrm{BH}}>M_{c} \\
0 & M_{\mathrm{BH}} \leqslant M_{c}
\end{array},\right.
$$

where $M_{c}$ is the minimum mass of a BH and $M_{\text {scale }}$ characterizes the width of the BH mass distribution. Özel et al. (2010) and Farr et al. (2011) present posterior distributions for $M_{c}$ and $M_{\text {scale }}$. Using the range of values in these distributions, we recompute the $\mathrm{BH}$ formation probability in the $P_{\mathrm{BH}, 1}$ limit (i.e., most stars with $M_{\text {ZAMS }}>40 M_{\odot}$ explode).

Altering the shape of the $\mathrm{BH}$ mass distribution, by varying $M_{c}$ and $M_{\text {scale }}$, changes where the $\mathrm{BH}$ formation probability function peaks. The statistical uncertainty in the $\mathrm{BH}$ mass distribution allows for peaks in $P_{\mathrm{BH}, 1}$ between ZAMS masses of $16.7 M_{\odot}$ and $21.2 M_{\odot}$, which amounts to an uncertainty of roughly $25 \%$ in the location of the peak. The width of the $\mathrm{BH}$ formation probability function changes significantly when we consider the statistical uncertainty in the BH mass distribution. The FWHM of the peak in $P_{\mathrm{BH}, 1}$ varies by an order of magnitude, ranging from $\sim 2 M_{\odot}$ to $20 M_{\odot}$. The large uncertainty in the extent of $P_{\mathrm{BH}}$ is a result of the poor constraints on the width of the $\mathrm{BH}$ mass distribution.

\subsection{The Connection of $P_{B H}$ to Stellar Structure}

We next investigate whether $P_{\mathrm{BH}}\left(M_{\mathrm{ZAMS}}\right)$ can be linked to a star's structure at collapse. O'Connor \& Ott (2011) investigated $\mathrm{BH}$ formation using hydrodynamic simulations. Their models suggested that the complex relationship between stellar structure and whether collapse would result in a successful SN explosion could be captured to first order by a single parameter, the compactness parameter

$$
\xi_{2.5}=\frac{2.5 M_{\odot}}{R\left(2.5 M_{\odot}\right) / 1000 \mathrm{~km}},
$$

where $R\left(2.5 M_{\odot}\right)$ is the radius that encloses $2.5 M_{\odot}$ at the time of core bounce (but see Ugliano et al. (2012) who showed that other aspects of the progenitor structure are important too). O'Connor \& Ott (2011), and later Ugliano et al. (2012), found that the neutrino mechanism generally failed to drive explosions in stars with large compactness parameters. These studies of the compactness parameter also suggest that there could be multiple, distinct ZAMS mass ranges that produce BHs because the relationship between $\xi_{2.5}$ and ZAMS mass is non-monotonic. The relationship between $\xi_{2.5}$ and $M_{\text {ZAMS }}$ was explored by Sukhbold \& Woosley (2014), who showed that the complicated mapping between these quantities is a result of the compactness parameter's sensitivity to not only the initial mass and composition of a star, but also the star's mixing and nuclear burning history.

To test whether $P_{\mathrm{BH}}$ is correlated with the compactness parameter, we plot $P_{\mathrm{BH}, 1}$ and $\xi_{2.5}$ as a function of ZAMS

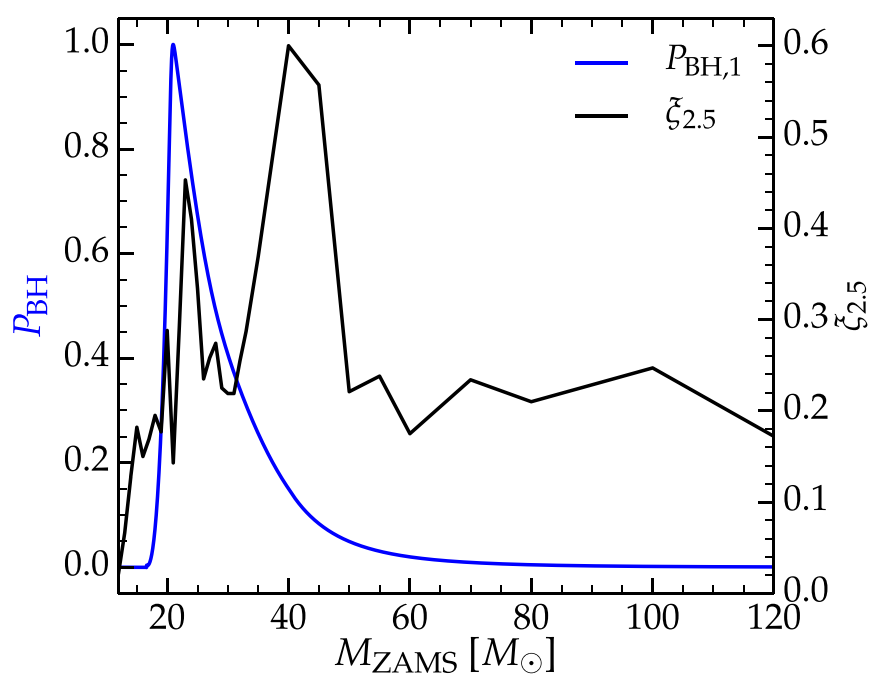

Figure 7. $\mathrm{BH}$ formation probability function $P_{\mathrm{BH}, 1}$ (blue, left axis) and compactness parameter (black, right axis) vs. ZAMS mass for the Woosley \& Heger (2007) pre-SN model set. O'Connor \& Ott (2011) argued that BH formation is most likely for stars with large values of $\xi_{2.5}$. There are two regions of high $\xi_{2.5}$, one near 22-25 $M_{\odot}$ and another near 35-45 $M_{\odot}$. The BH formation probability inferred from the observed $\mathrm{BH}$ mass distribution also peaks around $20 M_{\odot}$. The overlapping peaks in $\xi_{2.5}$ and $P_{\mathrm{BH}, 1}$ suggest that the $\mathrm{BH}$ formation probabilities computed in this work may have a physical origin related to the structure of the progenitor near the time of core collapse. However, there is not a peak in $P_{\mathrm{BH}, 1}$ that corresponds to the second peak in the compactness parameter near $40 M_{\odot}$.

mass in Figure 7. There is some agreement between $P_{\mathrm{BH}, 1}$ and $\xi_{2.5}$. Specifically, the peak in $P_{\mathrm{BH}}$ is coincident with the first peak in compactness. The similarity between $P_{\mathrm{BH}, 1}\left(M_{\mathrm{ZAMS}}\right)$ and $\xi_{2.5}\left(M_{\text {ZAMS }}\right)$ for $M_{\text {ZAMS }} \lesssim 35 M_{\odot}$ suggests that the observed $\mathrm{BH}$ mass distribution may be a manifestation of the fact that it is difficult, but not impossible, to explode stars with compact cores. In most situations, the stalled shock will not be revived in stars with large $\xi_{2.5}$, and they will collapse into BHs without explosion. However, on occasion, stochastic differences in the conditions at the onset of core collapse may permit successful explosions in otherwise identical stars.

The second, higher peak in compactness near $40 M_{\odot}$ does not appear to be echoed in $P_{\mathrm{BH}}$. Although there is a second peak in $P_{\mathrm{BH}, 2}$, it occurs at a much higher ZAMS mass of $\sim 60 \mathrm{M}_{\odot}$. There are several possible explanations for the absence of an appropriate second peak in $P_{\mathrm{BH}}$. First, if the observed sample of BHs is incomplete at the high mass end, then our models would underestimate the probability that stars with $M_{\text {ZAMS }} \sim 40 M_{\odot}$ produce $\mathrm{BHs}$. We explore this possibility by considering various levels of incompleteness above $10 M_{\odot}$ in the $\mathrm{BH}$ sample. Our tests show that a second peak in $P_{\mathrm{BH}}$ near $M_{\mathrm{ZAMS}}=35 M_{\odot}$ is recovered if the observed sample is less than $68 \%$ complete between $10-16 M_{\odot}$.

\subsubsection{The Impact of Extreme Mass Loss}

A second possibility is that the stellar evolution models used to compute $\xi_{2.5}$ do not adequately capture all of the physical processes that determine the core compactness. The Woosley \& Heger (2007) models include standard prescriptions to account for steady wind mass loss, but they do not consider extreme, eruptive mass loss. Massive stars, $\eta$ Car for example, are known to undergo outbursts that expel up to $20 M_{\odot}$ of the envelope on timescales of a decade (e.g., Smith \& Owocki 2006). These outbursts are thought to occur during the luminous blue variable 
Table 1

Core Property Changes Resulting from Envelope Removal

\begin{tabular}{lcccc}
\hline $\begin{array}{l}M_{\text {ZAMS }} \\
\left(M_{\odot}\right)\end{array}$ & $\begin{array}{c}\text { Evolutionary } \\
\text { Stage }\end{array}$ & $\begin{array}{c}\Delta M^{\mathrm{a}} \\
\left(M_{\odot}\right)\end{array}$ & $\begin{array}{c}\xi_{2.5} \text { Change }^{\mathrm{b}} \\
(\%)\end{array}$ & $\begin{array}{c}M_{\text {He core }} \text { Change } \\
(\%)\end{array}$ \\
\hline 35 & $\mathrm{HG}^{\mathrm{c}} / \mathrm{LBV}$ & 5.0 & -13 & -1.4 \\
35 & $\mathrm{HG} / \mathrm{LBV}$ & 10.0 & -12 & -5.0 \\
40 & $\mathrm{HG} / \mathrm{LBV}$ & 5.0 & -2 & -2.5 \\
40 & $\mathrm{HG} / \mathrm{LBV}$ & 10.0 & -11 & -17 \\
20 & $\mathrm{~GB}^{\mathrm{d}}$ & 12.78 & -7.9 & -11 \\
25 & GB & 14.45 & 12 & -25 \\
30 & GB & 15.27 & -7.1 & -28 \\
35 & GB & 16.03 & 11 & -42 \\
40 & GB & 15.36 & -17 & -35 \\
\hline
\end{tabular}

Notes.

a The amount of material removed.

b The value of $\xi_{2.5}$ was computed at the onset of core collapse, not at core bounce as in O'Connor \& Ott (2011).

c Hertzsprung gap.

d Giant branch.

(LBV) phase as the star transitions from the core hydrogen burning to core helium burning stages.

We use the one-dimensional stellar evolution code MESAstar, most recently described in Paxton et al. (2013), to assess the impact of such catastrophic mass loss on $\xi_{2.5}$. Our calculations assume the default parameter sets for massive star evolution included with version 6022 of MESAstar. The stars are evolved until they move across the Hertzsprung gap and reach the S Doradus instability strip (Wolf 1989). At this point we remove significant portions of the envelope by hand, and then continue evolving the stars to the onset of core collapse. Sukhbold \& Woosley (2014) showed that the compactness parameter is sensitive to slightly different implementations of stellar evolution physics. Therefore, it is not useful to directly compare the compactness parameters predicted by MESAstar to the values of $\xi_{2.5}$ shown in Figure 7, which were computed with the KEPLER code. Instead, we report relative values and trends in $\xi_{2.5}$ amongst the MESAstar models. The results are shown in Table 1, which lists the ZAMS mass of each star, the evolutionary state of the star when we removed the mass, the amount of material removed $\Delta M$, and the percent change of both $\xi_{2.5}$ and the mass of the helium core, relative to models without envelope removal.

For a star with solar metallicity and $M_{\mathrm{ZAMS}}=35 M_{\odot}$, removing $5 M_{\odot}$ and $10 M_{\odot}$ of the envelope reduces $\xi_{2.5}$ by $13 \%$ and $12 \%$, respectively, relative to a model without eruptive mass loss. Removing $5 M_{\odot}$ from a $40 M_{\odot}$ star while it is in the $\mathrm{S}$ Doradus instability strip only results in a $2 \%$ drop in the core compactness. In this case, stripping $10 M_{\odot}$ from the star lowers the compactness by $11 \%$. It appears that possible mass loss in LBV outbursts or other one-time or episodic processes will not significantly alter a star's core compactness or change the likelihood that it undergoes a successful SN explosion.

Additionally, the Woosley \& Heger (2007) single star evolution models do not account for mass loss triggered by interactions with a binary companion. All of the BHs that Ozel et al. (2010) used to construct the BH mass distribution are members of a binary. The standard formation channel for these BHXRTs involves a phase of common envelope evolution that drastically reduces the binary's orbital separation (van den Heuvel 1983). However, it is unclear how the low mass secondaries in BHXRTs are able to unbind the BH progenitor's massive envelopes before a merger occurs (e.g., Kalogera 1999; Podsiadlowski et al. 2003; Justham et al. 2006; Wiktorowicz et al. 2013). Accounting for heating from enhanced nuclear burning (Podsiadlowski et al. 2010) or the work done by the expanding envelope (Ivanova \& Chaichenets 2011) during the common envelope phase can balance the energy budget and allow these systems to avoid a merger. Because conventional BHXRT formation scenarios involve a phase of common envelope evolution, we examine how this phase will affect a star's final helium core mass and core compactness parameter.

We compute additional MESAstar models that mimic a phase of common envelope evolution that occurs after a star has crossed the Hertzsprung gap and a clear core-envelope boundary has been established (Ivanova \& Taam 2004; Belczynski et al. 2010). To accomplish this, we evolve five solar metallicity stars until a steep entropy gradient is established between the core and the convective envelope. We then remove the entire envelope and allow the stripped core to evolve until it begins to collapse. These stripped cores are compared to the cores produced in MESAstar models of stars that did not have their envelopes removed. The results of these comparisons are shown in Table 1.

For each star the core compactness $\xi_{2.5}$ changes by $\lesssim 17 \%$. We check whether the variation in $\xi_{2.5}$ is sensitive to the exact definition of the envelope by recomputing these models and moving the envelope boundary below or above the convective base. Models that assume deeper envelopes, which actually remove the outer layers of the core, exhibit the largest change in $\xi_{2.5}$. However the compactness does not deviate by more than $20 \%$ from that of a star that has not had its envelope removed.

On the other hand, the change in the helium core mass can be substantial. We find that the "post-common-envelope stars" have smaller helium cores than the unstripped stars, due to wind mass loss that occurs after the envelope is removed. The helium cores of the post-common-envelope stars fall in the range 5.67-10.62 $M_{\odot}$, compared to the range 6.36-16.52 $M_{\odot}$ for the unstripped stars. Although this mass range is narrower, it still spans the range of $\mathrm{BH}$ masses observed in BHXRTs, within the measurement errors.

Mass loss due to binary interactions is an additional source of stochasticity in the $M_{\mathrm{ZAMS}}-M_{\mathrm{BH}}$ relationship. In our MESAstar models of stars with $M_{\text {ZAMS }} \leqslant 30 M_{\odot}$, common envelope evolution results in a $\sim 10 \%-30 \%$ change in the helium core masses. This is comparable to the level of variation in the helium core masses stemming from different assumptions about metallicity and rotation in stellar evolution models (see Section 4.1). The helium core masses of the $35 M_{\odot}$ and $40 M_{\odot}$ post-commonenvelope stars are much smaller than the masses that we used to infer $P_{\mathrm{BH}}$. Since the observed $\mathrm{BH}$ mass function declines rapidly with increasing $M_{\mathrm{BH}}$, the larger helium core masses that we assumed would cause us to underestimate the number of 35-40 $M_{\odot}$ stars that produce BHs and, therefore, a value of $P_{\mathrm{BH}}$ that is too low. Thus, the lack of a second peak near $40 M_{\odot}$ in $P_{\mathrm{BH}, 1}$ and $P_{\mathrm{BH}, 2}$ could be a consequence of systematically smaller helium cores in the post-common-envelope $\mathrm{BH}$ progenitors in BHXRTs.

The stellar evolution models presented here suggest that extreme mass loss from evolved, massive stars will not significantly alter the final core compactness, and by extension the probability that a star will produce a $\mathrm{BH}$. The compactness is robust to this mass loss because the core becomes nearly isothermal once central hydrogen burning has ended. The thermal structure of the newly radiative core is insensitive to the pressure supplied by the envelope at its outer boundary. Thus, 
the envelope can be completely removed without disturbing the structure of the core. Because the trends in $\xi_{2.5}$ seen in our models have a clear physical origin, they can be trusted even though the version of MESAstar we used does not include the large nuclear network required to compute reliable values for the pre-SN compactness Sukhbold \& Woosley (2014).

\section{IMPLICATIONS AND CONCLUSIONS}

Motivated by the complicated relationship between $M_{\text {ZAMS }}$ and NS or $\mathrm{BH}$ formation, we have introduced a new paradigm for studying the final phases of a massive star's evolution: $a$ probabilistic description of $\mathrm{BH}$ formation. Using the $\mathrm{BH}$ mass distribution measured by Ozel et al. (2010), we have made a first exploration of the functional form of the $\mathrm{BH}$ formation probability function, $P_{\mathrm{BH}}\left(M_{\mathrm{ZAMS}}\right)$. Presently, the observational constraints on $P_{\mathrm{BH}}\left(M_{\mathrm{ZAMS}}\right)$ are too weak for us to make firm, quantitative predictions about its nature. Instead, we have illustrated the concept of probabilistic $\mathrm{BH}$ formation by deriving three possible forms of $P_{\mathrm{BH}}\left(M_{\mathrm{ZAMS}}\right)$ that are consistent with the weak constraints imposed by current measurements of the $\mathrm{BH}$ mass distribution and the level of chemical enrichment ascribed to massive stars. Although uncertain, the shapes of $P_{\mathrm{BH}}\left(M_{\mathrm{ZAMS}}\right)$ inferred here are suggestive of a link between the probability that a star produces a BH and its structure at the time of core collapse, as described by the compactness parameter $\xi_{2.5}$ (O'Connor \& Ott 2011). We have studied some of the complications in making this connection due to the effects of mass loss and binarity, which provide the first steps toward more detailed investigations in the future.

Our probabilistic description of $\mathrm{BH}$ formation is a substantial revision of the traditional ideas about which stars end their lives as NSs and which ultimately produce BHs. This new BH formation paradigm could potentially improve our understanding of and alter our expectations for the population of binary systems that harbor BHs and/or NSs. Including future, better constrained $\mathrm{BH}$ formation probability functions in binary population synthesis models may reveal new insights into the formation and evolution of $\mathrm{BH}$ X-ray binaries. Under the probabilistic $\mathrm{BH}$ formation scenario, the relative numbers of NS-NS, BH-NS, $\mathrm{BH}-\mathrm{BH}$ binaries, as well as the expected mass and mass ratio distributions amongst these binaries, could differ from the current predictions (see, e.g., Sipior \& Sigurdsson 2002; Belczynski et al. 2007; Sadowski et al. 2008; Abadie et al. 2010; Dominik et al. 2012).

Revised values that consider the effects of probabilistic $\mathrm{BH}$ formation have obvious implications for the expected gravitational wave signals and merger rates for the Advanced LIGO-Virgo detectors (Abbott et al. 2009; Accadia et al. 2012). Also, calculations that use a $\mathrm{BH}$ formation probability function may find increased formation rates for $\mathrm{BH}-$ millisecond pulsar binaries over previous studies (Sipior et al. 2004; Pfahl et al. 2005). In the $\mathrm{BH}$ formation probability function $P_{\mathrm{BH}, 1}$, the most massive stars, which have the shortest lifetimes, are likely to produce NSs instead of BHs. If these massive stars had a longer lived, $\sim 20 M_{\odot}$ companion, which according to $P_{\mathrm{BH}, 1}$ is likely to produce a $\mathrm{BH}$, it is possible for mass transfer from the companion to recycle the previously formed NS into a millisecond pulsar before this companion collapses.

The shape of the $\mathrm{BH}$ formation probability function will also determine the relative numbers of BHs and NSs, and a related quantity, the rate of unnovae. An unnova is when the observational signature of the birth of a $\mathrm{BH}$ is the disappearance of a star rather than a nova or SN-like brightening (e.g.,
Kochanek et al. 2008), although note that recent theoretical work argues that even these events will give rise to a low luminosity transient (Piro 2013; Lovegrove \& Woosley 2013). We demonstrate this by assuming that every star with $8 M_{\odot} \leqslant$ $M_{\text {ZAMS }} \leqslant 120 M_{\odot}$ produces either a NS or a BH at the end of its life, that the stars that produce NSs do so after a successful $\mathrm{SN}$ explosion, and that $\mathrm{BH}$ formation is not accompanied by a typical SN. Using our illustrative $\mathrm{BH}$ formation probability functions, $P_{\mathrm{BH}, 1}$ and $P_{\mathrm{BH}, 2}$, we find that the notional rate of unnovae is $\lesssim 10 \%-30 \%$ of the core collapse SN rate. For comparison, the traditional $\mathrm{BH}$ formation scenario with $M_{\mathrm{BH}}^{\star}=$ $25 M_{\odot}$ and the BH formation scenario proposed by Kochanek (2014) predict unnova rates of $25 \%$ and $\sim 20 \%$ of the core collapse SN rate, respectively. Measurements of the unnova rate could improve constraints on the traditional and probabilistic $\mathrm{BH}$ formation scenarios. Multiple observational surveys are capable of constraining the unnova rate. Kochanek et al. (2008) are conducting a search for "vanishing" stars that have collapsed into BHs without exploding. Additionally, high cadence optical surveys, like the Palomar Transient Factory (Rau et al. 2009), could identify the optical transient that may to accompany failed SNe (Lovegrove \& Woosley 2013; Piro 2013).

The authors acknowledge helpful exchanges with M. Cantiello, W. D. Arnett, S. de Mink, M. Renzo, S. Shore, U. C. T. Gamma, T. A. Thompson, and S. E. Woosley. We thank S. Couch, C. S. Kochanek, E. O'Connor, N. Smith, E. Lovegrove, J. F. Beacom, D. A. Perley, J. G. Cohen, E. N. Kirby, and T. Sukhbold for comments on a previous draft. C.D.O. states for the record that the idea of a black hole formation probability came to him after talking to Elizabeth Lovegrove at the 221st American Astronomical Society meeting at Long Beach, CA, in 2013 January. Lovegrove suggested to him that some $15 M_{\odot}$ stars might explode while other stars of the same initial mass might not. C.D.O. initially ridiculed the concept, but soon realized how wrong he was and that Lovegrove had an important point. This research is supported in part by NSF under grant numbers AST-1205732, AST-1212170, PHY-1151197, and PHY-1068881, by the Sherman Fairchild Foundation and the Sloan Foundation. The computations used resources of NSF's XSEDE network under allocation TG-PHY100033.

\section{REFERENCES}

Abadie, J., Abbott, B. P., Abbott, R., et al. 2010, CQGra, 27, 173001 Abbott, B. P., Abbott, R., Adhikari, R., et al. 2009, RPPh, 72, 076901 Accadia, T., Acernese, F., Alshourbagy, M., et al. 2012, JInst, 7, 3012 Bailyn, C. D., Jain, R. K., Coppi, P., \& Orosz, J. A. 1998, ApJ, 499, 367 Bastian, N., Covey, K. R., \& Meyer, M. R. 2010, ARA\&A, 48, 339 Belczynski, K., Dominik, M., Bulik, T., et al. 2010, ApJL, 715, L138 Belczynski, K., Taam, R. E., Kalogera, V., Rasio, F. A., \& Bulik, T. 2007, ApJ, 662,504

Belczynski, K., Wiktorowicz, G., Fryer, C. L., Holz, D. E., \& Kalogera, V. 2012, ApJ, 757, 91

Brown, J. M., \& Woosley, S. E. 2013, ApJ, 769, 99

Burrows, A. 1987, PhT, 40, 28

Couch, S. M., \& Ott, C. D. 2013, ApJL, 778, L7

Dessart, L., Livne, E., \& Waldman, R. 2010, MNRAS, 405, 2113

Dominik, M., Belczynski, K., Fryer, C., et al. 2012, ApJ, 759, 52

Eldridge, J. J., \& Tout, C. A. 2004, MNRAS, 353, 87

Farr, W. M., Sravan, N., Cantrell, A., et al. 2011, ApJ, 741, 103

Fryer, C. L. 1999, ApJ, 522, 413

Fryer, C. L., Belczynski, K., Wiktorowicz, G., et al. 2012, ApJ, 749, 91

Fryer, C. L., \& Kalogera, V. 2001, ApJ, 554, 548

Heger, A., Fryer, C. L., Woosley, S. E., Langer, N., \& Hartmann, D. H. 2003, ApJ, 591,288

Heger, A., Langer, N., \& Woosley, S. E. 2000, ApJ, 528, 368 
Ivanova, N., \& Chaichenets, S. 2011, ApJL, 731, L36

Ivanova, N., \& Taam, R. E. 2004, ApJ, 601, 1058

Justham, S., Rappaport, S., \& Podsiadlowski, P. 2006, MNRAS, 366, 1415

Kalogera, V. 1999, ApJ, 521, 723

Kochanek, C. S. 2014, ApJ, 785, 28

Kochanek, C. S., Beacom, J. F., Kistler, M. D., et al. 2008, ApJ, 684, 1336

Kreidberg, L., Bailyn, C. D., Farr, W. M., \& Kalogera, V. 2012, ApJ, 757, 36

Lovegrove, E., \& Woosley, S. E. 2013, ApJ, 769, 109

Maeder, A. 1992, A\&A, 264, 105

Meynet, G., \& Maeder, A. 2000, A\&A, 361, 101

Nadezhin, D. K. 1980, Ap\&SS, 69, 115

O’Connor, E., \& Ott, C. D. 2011, ApJ, 730, 70

Özel, F., Psaltis, D., Narayan, R., \& McClintock, J. E. 2010, ApJ, 725, 1918

Özel, F., Psaltis, D., Narayan, R., \& Santos Villarreal, A. 2012, ApJ, 757, 55

Paxton, B., Cantiello, M., Arras, P., et al. 2013, ApJS, 208, 4

Pejcha, O., Thompson, T. A., \& Kochanek, C. S. 2012, MNRAS, 424, 1570

Pfahl, E., Podsiadlowski, P., \& Rappaport, S. 2005, ApJ, 628, 343

Piro, A. L. 2013, ApJL, 768, L14

Podsiadlowski, P., Ivanova, N., Justham, S., \& Rappaport, S. 2010, MNRAS, 406, 840

Podsiadlowski, P., Rappaport, S., \& Han, Z. 2003, MNRAS, 341, 385

Rau, A., Kulkarni, S. R., Law, N. M., et al. 2009, PASP, 121, 1334
Remillard, R. A., \& McClintock, J. E. 2006, ARA\&A, 44, 49

Sadowski, A., Belczynski, K., Bulik, T., et al. 2008, ApJ, 676, 1162

Salpeter, E. E. 1955, ApJ, 121, 161

Shiode, J. H., \& Quataert, E. 2014, ApJ, 780, 96

Sipior, M. S., Portegies Zwart, S., \& Nelemans, G. 2004, MNRAS, 354, L49

Sipior, M. S., \& Sigurdsson, S. 2002, ApJ, 572, 962

Smartt, S. J., Eldridge, J. J., Crockett, R. M., \& Maund, J. R. 2009, MNRAS, 395,1409

Smith, N., \& Arnett, W. D. 2014, ApJ, 785, 82

Smith, N., Li, W., Filippenko, A. V., \& Chornock, R. 2011, MNRAS, 412, 1522

Smith, N., \& Owocki, S. P. 2006, ApJL, 645, L45

Sukhbold, T., \& Woosley, S. E. 2014, ApJ, 783, 10

Timmes, F. X., Woosley, S. E., \& Weaver, T. A. 1996, ApJ, 457, 834

Twarog, B. A., \& Wheeler, J. C. 1982, ApJ, 261, 636

Ugliano, M., Janka, H.-T., Marek, A., \& Arcones, A. 2012, ApJ, 757, 69

van den Heuvel, E. P. J. 1983, in Accretion-driven Stellar X-ray Sources, ed. W. H. G. Lewin \& E. P. J. van den Heuvel (Cambridge: Cambridge Univ. Press), 303

Wiktorowicz, G., Belczynski, K., \& Maccarone, T. J. 2013, arXiv:1312.5924

Wolf, B. 1989, A\&A, 217, 87

Woosley, S. E., \& Heger, A. 2007, PhR, 442, 269

Zhang, W., Woosley, S. E., \& Heger, A. 2008, ApJ, 679, 639 